\title{
Measurement of the $\tau^{-} \rightarrow K^{-} \pi^{0} \nu_{\tau}$ branching fraction
}

B. Aubert, ${ }^{1}$ M. Bona, ${ }^{1}$ D. Boutigny, ${ }_{1}^{1}$ Y. Karyotakis, ${ }^{1}$ J. P. Lees, ${ }^{1}$ V. Poireau,${ }^{1}$ X. Prudent, ${ }^{1}$ V. Tisserand,,${ }^{1}$ A. Zghiche, ${ }^{1}$ J. Garra Tico, ${ }^{2}$ E. Grauges ${ }^{2}$ L. Lopez, ${ }^{3}$ A. Palano, ${ }^{3}$ M. Pappagallo, ${ }^{3}$ G. Eigen, ${ }^{4}$ B. Stugu, ${ }^{4}$ L. Sun, ${ }^{4}$ G. S. Abrams, ${ }^{5}$ M. Battaglia, ${ }^{5}$ D. N. Brown, ${ }^{5}$ J. Button-Shafer, ${ }^{5}$ R. N. Cahn, ${ }^{5}$ Y. Groysman, ${ }^{5}$ R. G. Jacobsen, ${ }^{5}$ J. A. Kadyk, ${ }^{5}$ L. T. Kerth, ${ }^{5}$ Yu. G. Kolomensky, ${ }^{5}$ G. Kukartsev, ${ }^{5}$ D. Lopes Pegna, ${ }^{5}$ G. Lynch, ${ }^{5}$ L. M. Mir, ${ }^{5}$ T. J. Orimoto, ${ }^{5}$ I. L. Osipenkov, ${ }^{5}$ M. T. Ronan, ${ }^{5, *}$ K. Tackmann, ${ }^{5}$ T. Tanabe, ${ }^{5}$ W. A. Wenzel, ${ }^{5}$ P. del Amo Sanchez, ${ }^{6}$ C. M. Hawkes, ${ }^{6}$ A. T. Watson, ${ }^{6}$ T. Held, ${ }^{7}$ H. Koch, ${ }^{7}$ M. Pelizaeus, ${ }^{7}$ T. Schroeder, ${ }^{7}$ M. Steinke, ${ }^{7}$ D. Walker, ${ }^{8}$ D. J. Asgeirsson, ${ }^{9}$ T. Cuhadar-Donszelmann, ${ }^{9}$ B. G. Fulsom, ${ }^{9}$ C. Hearty, ${ }^{9}$ T. S. Mattison, ${ }^{9}$ J. A. McKenna, ${ }^{9}$ A. Khan, ${ }^{10}$ M. Saleem, ${ }^{10}$ L. Teodorescu, ${ }^{10}$ V. E. Blinov, ${ }^{11}$ A. D. Bukin, ${ }^{11}$ V. P. Druzhinin, ${ }^{11}$ V. B. Golubev, ${ }^{11}$ A. P. Onuchin, ${ }^{11}$ S. I. Serednyakov, ${ }^{11}$ Yu. I. Skovpen, ${ }^{11}$ E. P. Solodov, ${ }^{11}$ K. Yu. Todyshev, ${ }^{11}$ M. Bondioli, ${ }^{12}$ S. Curry, ${ }^{12}$ I. Eschrich, ${ }^{12}$ D. Kirkby, ${ }^{12}$ A. J. Lankford, ${ }^{12}$ P. Lund, ${ }^{12}$ M. Mandelkern, ${ }^{12}$ E. C. Martin, ${ }^{12}$ D. P. Stoker, ${ }^{12}$ S. Abachi,${ }^{13}$ C. Buchanan,${ }^{13}$ S. D. Foulkes, ${ }^{14}$ J. W. Gary, ${ }^{14}$ F. Liu, ${ }^{14}$ O. Long,,${ }^{14}$ B. C. Shen,${ }^{14}$ L. Zhang, ${ }^{14}$ H. P. Paar, ${ }^{15}$ S. Rahatlou, ${ }^{15}$ V. Sharma, ${ }^{15}$ J. W. Berryhill, ${ }^{16}$ C. Campagnari, ${ }^{16}$ A. Cunha, ${ }^{16}$ B. Dahmes, ${ }^{16}$

T. M. Hong, ${ }^{16}$ D. Kovalskyi, ${ }^{16}$ J. D. Richman, ${ }^{16}$ T. W. Beck, ${ }^{17}$ A. M. Eisner, ${ }^{17}$ C. J. Flacco, ${ }^{17}$ C. A. Heusch, ${ }^{17}$

J. Kroseberg, ${ }^{17}$ W. S. Lockman, ${ }^{17}$ T. Schalk, ${ }^{17}$ B. A. Schumm, ${ }^{17}$ A. Seiden, ${ }^{17}$ M. G. Wilson, ${ }^{17}$ L. O. Winstrom, ${ }^{17}$ E. Chen, ${ }^{18}$ C. H. Cheng, ${ }^{18}$ F. Fang, ${ }^{18}$ D. G. Hitlin, ${ }^{18}$ I. Narsky, ${ }^{18}$ T. Piatenko, ${ }^{18}$ F. C. Porter, ${ }^{18}$ R. Andreassen, ${ }^{19}$ G. Mancinelli, ${ }^{19}$ B. T. Meadows, ${ }^{19}$ K. Mishra, ${ }^{19}$ M. D. Sokoloff, ${ }^{19}$ F. Blanc, ${ }^{20}$ P. C. Bloom, ${ }^{20}$ S. Chen, ${ }^{20}$ W. T. Ford, ${ }^{20}$ J. F. Hirschauer, ${ }^{20}$ A. Kreisel,${ }^{20}$ M. Nagel, ${ }^{20}$ U. Nauenberg, ${ }^{20}$ A. Olivas, ${ }^{20}$ J. G. Smith,${ }^{20}$ K. A. Ulmer, ${ }^{20}$ S. R. Wagner, ${ }^{20}$ J. Zhang, ${ }^{20}$ A. M. Gabareen, ${ }^{21}$ A. Soffer, ${ }^{21, \dagger}$ W. H. Toki, ${ }^{21}$ R. J. Wilson, ${ }^{21}$ F. Winklmeier,${ }^{21}$ D. D. Altenburg, ${ }^{22}$ E. Feltresi, ${ }^{22}$ A. Hauke, ${ }^{22}$ H. Jasper, ${ }^{22}$ J. Merkel, ${ }^{22}$ A. Petzold, ${ }^{22}$ B. Spaan, ${ }^{22}$ K. Wacker, ${ }^{22}$ V. Klose, ${ }^{23}$ M. J. Kobel, ${ }^{23}$ H. M. Lacker ${ }^{23}$ W. F. Mader, ${ }^{23}$ R. Nogowski, ${ }^{23}$ J. Schubert, ${ }^{23}$ K. R. Schubert, ${ }^{23}$ R. Schwierz, ${ }^{23}$ J. E. Sundermann, ${ }^{23}$ A. Volk, ${ }^{23}$

D. Bernard, ${ }^{24}$ G. R. Bonneaud, ${ }^{24}$ E. Latour, ${ }^{24}$ V. Lombardo,${ }^{24}$ Ch. Thiebaux, ${ }^{24}$ M. Verderi, ${ }^{24}$ P. J. Clark, ${ }^{25}$ W. Gradl, ${ }^{25}$ F. Muheim, ${ }^{25}$ S. Playfer ${ }^{25}$ A. I. Robertson, ${ }^{25}$ J. E. Watson, ${ }^{25}$ Y. Xie, ${ }^{25}$ M. Andreotti, ${ }^{26}$ D. Bettoni, ${ }^{26}$ C. Bozzi, ${ }^{26}$ R. Calabrese, ${ }^{26}$ A. Cecchi, ${ }^{26}$ G. Cibinetto, ${ }^{26}$ P. Franchini, ${ }^{26}$ E. Luppi, ${ }^{26}$ M. Negrini, ${ }^{26}$ A. Petrella, ${ }^{26}$ L. Piemontese, ${ }^{26}$ E. Prencipe ${ }^{26}$ V. Santoro, ${ }^{26}$ F. Anulli ${ }^{27}$ R. Baldini-Ferroli, ${ }^{27}$ A. Calcaterra ${ }^{27}$ R. de Sangro, ${ }^{27}$ G. Finocchiaro, ${ }^{27}$ S. Pacetti, ${ }^{27}$ P. Patteri, ${ }^{27}$ I. M. Peruzzi, ${ }^{27,}$ M. Piccolo, ${ }^{27}$ M. Rama,${ }^{27}$ A. Zallo, ${ }^{27}$ A. Buzzo,${ }^{28}$ R. Contri, ${ }^{28}$ M. Lo Vetere,${ }^{28}$ M. M. Macri, ${ }^{28}$ M. R. Monge, ${ }^{28}$ S. Passaggio, ${ }^{28}$ C. Patrignani, ${ }^{28}$ E. Robutti, ${ }^{28}$ A. Santroni,${ }^{28}$ S. Tosi, ${ }^{28}$

K. S. Chaisanguanthum, ${ }^{29}$ M. Morii, ${ }^{29}$ J. Wu ${ }^{29}$ R. S. Dubitzky, ${ }^{30}$ J. Marks,${ }^{30}$ S. Schenk, ${ }^{30}$ U. Uwer, ${ }^{30}$ D. J. Bard, ${ }^{31}$ P. D. Dauncey, ${ }^{31}$ R. L. Flack, ${ }^{31}$ J. A. Nash, ${ }^{31}$ W. Panduro Vazquez, ${ }^{31}$ M. Tibbetts, ${ }^{31}$ P. K. Behera, ${ }^{32}$ X. Chai, ${ }^{32}$

M. J. Charles, ${ }^{32}$ U. Mallik, ${ }^{32}$ V. Ziegler, ${ }^{32}$ J. Cochran, ${ }^{33}$ H. B. Crawley, ${ }^{33}$ L. Dong, ${ }^{33}$ V. Eyges, ${ }^{33}$ W. T. Meyer, ${ }^{33}$ S. Prell, ${ }^{33}$

E. I. Rosenberg, ${ }^{33}$ A. E. Rubin, ${ }^{33}$ Y. Y. Gao, ${ }^{34}$ A. V. Gritsan, ${ }^{34}$ Z. J. Guo, ${ }^{34}$ C. K. Lae, ${ }^{34}$ A. G. Denig, ${ }^{35}$ M. Fritsch, ${ }^{35}$ G. Schott, ${ }^{35}$ N. Arnaud, ${ }^{36}$ J. Béquilleux, ${ }^{36}$ A. D'Orazio, ${ }^{36}$ M. Davier ${ }^{36}$ G. Grosdidier, ${ }^{36}$ A. Höcker, ${ }^{36}$ V. Lepeltier, ${ }^{36}$

F. Le Diberder, ${ }^{36}$ A. M. Lutz, ${ }^{36}$ S. Pruvot, ${ }^{36}$ S. Rodier, ${ }^{36}$ P. Roudeau, ${ }^{36}$ M. H. Schune, ${ }^{36}$ J. Serrano, ${ }^{36}$ V. Sordini, ${ }^{36}$ A. Stocchi, ${ }^{36}$ W. F. Wang, ${ }^{36}$ G. Wormser, ${ }^{36}$ D. J. Lange, ${ }^{37}$ D. M. Wright, ${ }^{37}$ I. Bingham, ${ }^{38}$ C. A. Chavez, ${ }^{38}$ I. J. Forster, ${ }^{38}$ J. R. Fry, ${ }^{38}$ E. Gabathuler, ${ }^{38}$ R. Gamet, ${ }^{38}$ D. E. Hutchcroft, ${ }^{38}$ D. J. Payne, ${ }^{38}$ K. C. Schofield, ${ }^{38}$ C. Touramanis, ${ }^{38}$ A. J. Bevan, ${ }^{39}$ K. A. George, ${ }^{39}$ F. Di Lodovico, ${ }^{39}$ W. Menges,${ }^{39}$ R. Sacco, ${ }^{39}$ G. Cowan, ${ }^{40}$ H. U. Flaecher, ${ }^{40}$ D. A. Hopkins, ${ }^{40}$ S. Paramesvaran, ${ }^{40}$ F. Salvatore, ${ }^{40}$ A. C. Wren,${ }^{40}$ D. N. Brown, ${ }^{41}$ C. L. Davis,${ }^{41}$ J. Allison, ${ }^{42}$ N. R. Barlow, ${ }^{42}$ R. J. Barlow, ${ }^{42}$ Y. M. Chia, ${ }^{42}$ C. L. Edgar, ${ }^{42}$ G. D. Lafferty, ${ }^{42}$ T. J. West, ${ }^{42}$ J. I. Yi, ${ }^{42}$ J. Anderson, ${ }^{43}$ C. Chen, ${ }^{43}$ A. Jawahery, ${ }^{43}$

D. A. Roberts, ${ }^{43}$ G. Simi, ${ }^{43}$ J. M. Tuggle, ${ }^{43}$ G. Blaylock, ${ }^{44}$ C. Dallapiccola, ${ }^{44}$ S. S. Hertzbach, ${ }^{44}$ X. Li,${ }^{44}$ T. B. Moore ${ }^{44}$ E. Salvati, ${ }^{44}$ S. Saremi, ${ }^{44}$ R. Cowan, ${ }^{45}$ D. Dujmic, ${ }^{45}$ P. H. Fisher, ${ }^{45}$ K. Koeneke, ${ }^{45}$ G. Sciolla, ${ }^{45}$ S. J. Sekula, ${ }^{45}$

M. Spitznagel, ${ }^{45}$ F. Taylor, ${ }^{45}$ R. K. Yamamoto, ${ }^{45}$ M. Zhao, ${ }^{45}$ Y. Zheng, ${ }^{45}$ S. E. Mclachlin, ${ }^{46, *}$ P. M. Patel, ${ }^{46}$ S. H. Robertson, ${ }^{46}$ A. Lazzaro, ${ }^{47}$ F. Palombo, ${ }^{47}$ J. M. Bauer, ${ }^{48}$ L. Cremaldi, ${ }^{48}$ V. Eschenburg, ${ }^{48}$ R. Godang, ${ }^{48}$ R. Kroeger,${ }^{48}$ D. A. Sanders, ${ }^{48}$ D. J. Summers, ${ }^{48}$ H. W. Zhao, ${ }^{48}$ S. Brunet, ${ }^{49}$ D. Côté, ${ }^{49}$ M. Simard, ${ }^{49}$ P. Taras, ${ }^{49}$ F. B. Viaud, ${ }^{49}$ H. Nicholson, ${ }^{50}$ G. De Nardo, ${ }^{51}$ F. Fabozzi, ${ }^{51,8}$ L. Lista, ${ }^{51}$ D. Monorchio, ${ }^{51}$ C. Sciacca, ${ }^{51}$ M. A. Baak, ${ }^{52}$ G. Raven,${ }^{52}$ H. L. Snoek, ${ }^{52}$ C. P. Jessop, ${ }^{53}$ K. J. Knoepfel,${ }^{53}$ J. M. LoSecco, ${ }^{53}$ G. Benelli, ${ }^{54}$ L. A. Corwin,${ }^{54}$ K. Honscheid,${ }^{54}$ H. Kagan, ${ }^{54}$ R. Kass,${ }^{54}$ J. P. Morris,${ }^{54}$ A. M. Rahimi,${ }^{54}$ J. J. Regensburger, ${ }^{54}$ Q. K. Wong,${ }^{54}$ N. L. Blount, ${ }^{55}$ J. Brau,${ }^{55}$ R. Frey, ${ }^{55}$ O. Igonkina, ${ }^{55}$ J. A. Kolb, ${ }^{55}$ M. Lu, ${ }^{55}$ R. Rahmat, ${ }^{55}$ N. B. Sinev, ${ }^{55}$ D. Strom,${ }^{55}$ J. Strube,${ }^{55}$ E. Torrence, ${ }^{55}$ N. Gagliardi ${ }^{56}$ A. Gaz ${ }^{56}$ M. Margoni, ${ }^{56}$ M. Morandin, ${ }^{56}$ A. Pompili, ${ }^{56}$ M. Posocco,${ }^{56}$ M. Rotondo, ${ }^{56}$ F. Simonetto, ${ }^{56}$ R. Stroili, ${ }^{56}$ C. Voci, ${ }^{56}$ E. Ben-Haim, ${ }^{57}$ H. Briand,${ }^{57}$ G. Calderini, ${ }^{57}$ J. Chauveau, ${ }^{57}$ P. David,${ }^{57}$ L. Del Buono, ${ }^{57}$ Ch. de la Vaissière,${ }^{57}$ O. Hamon, ${ }^{57} \mathrm{Ph}$. Leruste, ${ }^{57} \mathrm{~J}$. Malclès, ${ }^{57} \mathrm{~J}$. Ocariz,${ }^{57}$ A. Perez, ${ }^{57} \mathrm{~J}$. Prendki, ${ }^{57}$ L. Gladney, ${ }^{58}$ M. Biasini,,${ }^{59}$ R. Covarelli, ${ }^{59}$ E. Manoni, ${ }^{59}$ C. Angelini, ${ }^{60}$ G. Batignani, ${ }^{60}$ S. Bettarini, ${ }^{60}$ M. Carpinelli, ${ }^{60}$ R. Cenci, ${ }^{60}$ A. Cervelli, ${ }^{60}$ F. Forti, ${ }^{60}$ 
M. A. Giorgi, ${ }^{60}$ A. Lusiani, ${ }^{60}$ G. Marchiori, ${ }^{60}$ M. A. Mazur, ${ }^{60}$ M. Morganti, ${ }^{60}$ N. Neri, ${ }^{60}$ E. Paoloni, ${ }^{60}$ G. Rizzo, ${ }^{60}$ J. J. Walsh ${ }^{60}$ M. Haire, ${ }^{61}$ J. Biesiada, ${ }^{62}$ P. Elmer, ${ }^{62}$ Y. P. Lau, ${ }^{62}$ C. Lu ${ }^{62}$ J. Olsen, ${ }^{62}$ A. J. S. Smith, ${ }^{62}$ A. V. Telnov, ${ }^{62}$ E. Baracchini, ${ }^{63}$ F. Bellini, ${ }^{63}$ G. Cavoto, ${ }^{63}$ D. del Re, ${ }^{63}$ E. Di Marco, ${ }^{63}$ R. Faccini, ${ }^{63}$ F. Ferrarotto, ${ }^{63}$ F. Ferroni, ${ }^{63}$ M. Gaspero, ${ }^{63}$ P. D. Jackson, ${ }^{63}$ L. Li Gioi ${ }^{63}$ M. A. Mazzoni, ${ }^{63}$ S. Morganti ${ }^{63}$ G. Piredda, ${ }^{63}$ F. Polci, ${ }^{63}$ F. Renga, ${ }^{63}$ C. Voena, ${ }^{63}$ M. Ebert, ${ }^{64}$ T. Hartmann, ${ }^{64}$ H. Schröder, ${ }^{64}$ R. Waldi, ${ }^{64}$ T. Adye, ${ }^{65}$ G. Castelli, ${ }^{65}$ B. Franek, ${ }^{65}$ E. O. Olaiya, ${ }^{65}$ S. Ricciardi, ${ }^{65}$ W. Roethel, ${ }^{65}$ F. F. Wilson, ${ }^{65}$ S. Emery, ${ }^{66}$ M. Escalier, ${ }^{66}$ A. Gaidot, ${ }^{66}$ S. F. Ganzhur, ${ }^{66}$ G. Hamel de Monchenault, ${ }^{66}$ W. Kozanecki, ${ }^{66}$ G. Vasseur, ${ }^{66}$ Ch. Yèche, ${ }^{66}$ M. Zito, ${ }^{66}$ X. R. Chen,${ }^{67}$ H. Liu, ${ }^{67}$ W. Park, ${ }^{67}$ M. V. Purohit, ${ }^{67}$ J. R. Wilson, ${ }^{67}$ M. T. Allen, ${ }^{68}$ D. Aston, ${ }^{68}$ R. Bartoldus, ${ }^{68}$ P. Bechtle, ${ }^{68}$ N. Berger, ${ }^{68}$ R. Claus, ${ }^{68}$ J. P. Coleman, ${ }^{68}$ M. R. Convery ${ }^{68}$ J. C. Dingfelder, ${ }^{68}$ J. Dorfan, ${ }^{68}$ G. P. Dubois-Felsmann, ${ }^{68}$ W. Dunwoodie, ${ }^{68}$ R. C. Field ${ }^{68}$ T. Glanzman, ${ }^{68}$ S. J. Gowdy, ${ }^{68}$ M. T. Graham, ${ }^{68}$ P. Grenier ${ }^{68}$ C. Hast ${ }^{68}$ T. Hryn'ova, ${ }^{68}$ W. R. Innes, ${ }^{68}$ J. Kaminski, ${ }^{68}$ M. H. Kelsey, ${ }^{68}$ H. Kim, ${ }^{68}$ P. Kim, ${ }^{68}$ M. L. Kocian, ${ }^{68}$ D. W. G. S. Leith, ${ }^{68}$ S. Li, ${ }^{68}$ S. Luitz, ${ }^{68}$ V. Luth, ${ }^{68}$ H. L. Lynch, ${ }^{68}$ D. B. MacFarlane, ${ }^{68}$ H. Marsiske, ${ }^{68}$ R. Messner, ${ }^{68}$ D. R. Muller ${ }^{68}$ C. P. O' Grady, ${ }^{68}$ I. Ofte, ${ }^{68}$ A. Perazzo,${ }^{68}$ M. Perl, ${ }^{68}$ T. Pulliam, ${ }^{68}$ B. N. Ratcliff, ${ }^{68}$ A. Roodman, ${ }^{68}$ A. A. Salnikov, ${ }^{68}$ R. H. Schindler, ${ }^{68}$ J. Schwiening, ${ }^{68}$ A. Snyder, ${ }^{68}$ J. Stelzer, ${ }^{68}$ D. Su, ${ }^{68}$ M. K. Sullivan, ${ }^{68}$ K. Suzuki, ${ }^{68}$ S. K. Swain, ${ }^{68}$ J. M. Thompson, ${ }^{68}$ J. Va'vra, ${ }^{68}$ N. van Bakel, ${ }^{68}$ A. P. Wagner, ${ }^{68}$ M. Weaver, ${ }^{68}$ W. J. Wisniewski, ${ }^{68}$ M. Wittgen, ${ }^{68}$ D. H. Wright, ${ }^{68}$ A. K. Yarritu ${ }^{68}$ K. Yi, ${ }^{68}$ C. C. Young, ${ }^{68}$ P. R. Burchat, ${ }^{69}$ A. J. Edwards, ${ }^{69}$ S. A. Majewski, ${ }^{69}$ B. A. Petersen, ${ }^{69}$ L. Wilden, ${ }^{69}$ S. Ahmed, ${ }^{70}$ M. S. Alam, ${ }^{70}$ R. Bula, ${ }^{70}$ J. A. Ernst ${ }^{70}$ V. Jain, ${ }^{70}$ B. Pan, ${ }^{70}$ M. A. Saeed, ${ }^{70}$ F. R. Wappler, ${ }^{70}$ S. B. Zain, ${ }^{70}$ M. Krishnamurthy, ${ }^{71}$ S. M. Spanier ${ }^{71}$ R. Eckmann, ${ }^{72}$ J. L. Ritchie, ${ }^{72}$ A. M. Ruland, ${ }^{72}$ C. J. Schilling, ${ }^{72}$ R. F. Schwitters, ${ }^{72}$ J. M. Izen, ${ }^{73}$ X. C. Lou, ${ }^{73}$ S. Ye, ${ }^{73}$ F. Bianchi, ${ }^{74}$ F. Gallo, ${ }^{74}$ D. Gamba,${ }^{74}$ M. Pelliccioni, ${ }^{74}$ M. Bomben,${ }^{75}$ L. Bosisio,${ }^{75}$ C. Cartaro, ${ }^{75}$ F. Cossutti, ${ }^{75}$ G. Della Ricca, ${ }^{75}$ L. Lanceri, ${ }^{75}$ L. Vitale, ${ }^{75}$ V. Azzolini ${ }^{76}$ N. Lopez-March, ${ }^{76}$ F. Martinez-Vidal,${ }^{76, \mid l}$ D. A. Milanes, ${ }^{76}$ A. Oyanguren, ${ }^{76}$ J. Albert, ${ }^{77}$ Sw. Banerjee,${ }^{77}$ B. Bhuyan, ${ }^{77}$ K. Hamano, ${ }^{77}$ R. Kowalewski, ${ }^{77}$ I. M. Nugent, ${ }^{77}$ J. M. Roney, ${ }^{77}$ R. J. Sobie,${ }^{77}$ P. F. Harrison, ${ }^{78}$ J. Ilic, ${ }^{78}$ T. E. Latham,${ }^{78}$ G. B. Mohanty, ${ }^{78}$ H. R. Band,${ }^{79}$ X. Chen, ${ }^{79}$ S. Dasu, ${ }^{79}$ K. T. Flood, ${ }^{79}$ J. J. Hollar, ${ }^{79}$ P. E. Kutter, ${ }^{79}$ Y. Pan, ${ }^{79}$ M. Pierini, ${ }^{79}$ R. Prepost, ${ }^{79}$ S. L. Wu, ${ }^{79}$ and H. Neal ${ }^{80}$

\section{(BABAR Collaboration)}

\footnotetext{
${ }^{1}$ Laboratoire de Physique des Particules, IN2P3/CNRS et Université de Savoie, F-74941 Annecy-Le-Vieux, France

${ }^{2}$ Universitat de Barcelona, Facultat de Fisica, Departament ECM, E-08028 Barcelona, Spain

${ }^{3}$ Università di Bari, Dipartimento di Fisica and INFN, I-70126 Bari, Italy

${ }^{4}$ University of Bergen, Institute of Physics, N-5007 Bergen, Norway

${ }^{5}$ Lawrence Berkeley National Laboratory and University of California, Berkeley, California 94720, USA

${ }^{6}$ University of Birmingham, Birmingham, B15 2TT, United Kingdom

${ }^{7}$ Ruhr Universität Bochum, Institut für Experimentalphysik 1, D-44780 Bochum, Germany

${ }^{8}$ University of Bristol, Bristol BS8 1TL, United Kingdom

${ }^{9}$ University of British Columbia, Vancouver, British Columbia, Canada V6T 1 Z1

${ }^{10}$ Brunel University, Uxbridge, Middlesex UB8 3PH, United Kingdom

${ }^{11}$ Budker Institute of Nuclear Physics, Novosibirsk 630090, Russia

${ }^{12}$ University of California at Irvine, Irvine, California 92697, USA

${ }^{13}$ University of California at Los Angeles, Los Angeles, California 90024, USA

${ }^{14}$ University of California at Riverside, Riverside, California 92521, USA

${ }^{15}$ University of California at San Diego, La Jolla, California 92093, USA

${ }^{16}$ University of California at Santa Barbara, Santa Barbara, California 93106, USA

${ }^{17}$ University of California at Santa Cruz, Institute for Particle Physics, Santa Cruz, California 95064, USA

${ }^{18}$ California Institute of Technology, Pasadena, California 91125, USA

${ }^{19}$ University of Cincinnati, Cincinnati, Ohio 45221, USA

${ }^{20}$ University of Colorado, Boulder, Colorado 80309, USA

${ }^{21}$ Colorado State University, Fort Collins, Colorado 80523, USA

${ }^{22}$ Universität Dortmund, Institut für Physik, D-44221 Dortmund, Germany

${ }^{23}$ Technische Universität Dresden, Institut für Kernund Teilchenphysik, D-01062 Dresden, Germany

${ }^{24}$ Laboratoire Leprince-Ringuet, CNRS/IN2P3, Ecole Polytechnique, F-91128 Palaiseau, France

${ }^{25}$ University of Edinburgh, Edinburgh EH9 3JZ, United Kingdom

${ }^{26}$ Università di Ferrara, Dipartimento di Fisica and INFN, I-44100 Ferrara, Italy

${ }^{27}$ Laboratori Nazionali di Frascati dell'INFN, I-00044 Frascati, Italy

${ }^{28}$ Università di Genova, Dipartimento di Fisica and INFN, I-16146 Genova, Italy

${ }^{29}$ Harvard University, Cambridge, Massachusetts 02138, USA

${ }^{30}$ Universität Heidelberg, Physikalisches Institut, Philosophenweg 12, D-69120 Heidelberg, Germany
} 
${ }^{31}$ Imperial College London, London, SW7 2AZ, United Kingdom

${ }^{32}$ University of Iowa, Iowa City, Iowa 52242, USA

${ }^{33}$ Iowa State University, Ames, Iowa 50011-3160, USA

${ }^{34}$ Johns Hopkins University, Baltimore, Maryland 21218, USA

${ }^{35}$ Universität Karlsruhe, Institut für Experimentelle Kernphysik, D-76021 Karlsruhe, Germany

${ }^{36}$ Laboratoire de l'Accélérateur Linéaire, IN2P3/CNRS et Université Paris-Sud 11, Centre Scientifique d'Orsay, B. P. 34, F-91898 ORSAY Cedex, France

${ }^{37}$ Lawrence Livermore National Laboratory, Livermore, California 94550, USA

${ }^{38}$ University of Liverpool, Liverpool L69 7ZE, United Kingdom

${ }^{39}$ Queen Mary, University of London, E1 4NS, United Kingdom

${ }^{40}$ University of London, Royal Holloway and Bedford New College, Egham, Surrey TW20 OEX, United Kingdom

${ }^{41}$ University of Louisville, Louisville, Kentucky 40292, USA

${ }^{42}$ University of Manchester, Manchester M13 9PL, United Kingdom

${ }^{43}$ University of Maryland, College Park, Maryland 20742, USA

${ }^{44}$ University of Massachusetts, Amherst, Massachusetts 01003, USA

${ }^{45}$ Massachusetts Institute of Technology, Laboratory for Nuclear Science, Cambridge, Massachusetts 02139, USA

${ }^{46}$ McGill University, Montréal, Québec, Canada H3A 2T8

${ }^{47}$ Università di Milano, Dipartimento di Fisica and INFN, I-20133 Milano, Italy

${ }^{48}$ University of Mississippi, University, Mississippi 38677, USA

${ }^{49}$ Université de Montréal, Physique des Particules, Montréal, Québec, Canada H3C 3J7

${ }^{50}$ Mount Holyoke College, South Hadley, Massachusetts 01075, USA

${ }^{51}$ Università di Napoli Federico II, Dipartimento di Scienze Fisiche and INFN, I-80126, Napoli, Italy

${ }^{52}$ NIKHEF, National Institute for Nuclear Physics and High Energy Physics, NL-1009 DB Amsterdam, The Netherlands

${ }^{53}$ University of Notre Dame, Notre Dame, Indiana 46556, USA

${ }^{54}$ Ohio State University, Columbus, Ohio 43210, USA

${ }^{55}$ University of Oregon, Eugene, Oregon 97403, USA

${ }^{56}$ Università di Padova, Dipartimento di Fisica and INFN, I-35131 Padova, Italy

${ }^{57}$ Laboratoire de Physique Nucléaire et de Hautes Energies, IN2P3/CNRS, Université Pierre et Marie Curie-Paris6, Université Denis Diderot-Paris7, F-75252 Paris, France

${ }^{58}$ University of Pennsylvania, Philadelphia, Pennsylvania 19104, USA

${ }^{59}$ Università di Perugia, Dipartimento di Fisica and INFN, I-06100 Perugia, Italy

${ }^{60}$ Università di Pisa, Dipartimento di Fisica, Scuola Normale Superiore and INFN, I-56127 Pisa, Italy

${ }^{61}$ Prairie View A\&M University, Prairie View, Texas 77446, USA

${ }^{62}$ Princeton University, Princeton, New Jersey 08544, USA

${ }^{63}$ Università di Roma La Sapienza, Dipartimento di Fisica and INFN, I-00185 Roma, Italy

${ }^{64}$ Universität Rostock, D-18051 Rostock, Germany

${ }^{65}$ Rutherford Appleton Laboratory, Chilton, Didcot, Oxon, OX11 0QX, United Kingdom

${ }^{66}$ DSM/Dapnia, CEA/Saclay, F-91191 Gif-sur-Yvette, France

${ }^{67}$ University of South Carolina, Columbia, South Carolina 29208, USA

${ }^{68}$ Stanford Linear Accelerator Center, Stanford, California 94309, USA

${ }^{69}$ Stanford University, Stanford, California 94305-4060, USA

${ }^{70}$ State University of New York, Albany, New York 12222, USA

${ }^{71}$ University of Tennessee, Knoxville, Tennessee 37996, USA

${ }^{72}$ University of Texas at Austin, Austin, Texas 78712, USA

${ }^{73}$ University of Texas at Dallas, Richardson, Texas 75083, USA

${ }^{74}$ Università di Torino, Dipartimento di Fisica Sperimentale and INFN, I-10125 Torino, Italy

${ }^{75}$ Università di Trieste, Dipartimento di Fisica and INFN, I-34127 Trieste, Italy

${ }^{76}$ IFIC, Universitat de Valencia-CSIC, E-46071 Valencia, Spain

${ }^{77}$ University of Victoria, Victoria, British Columbia, Canada V8W 3P6

${ }^{78}$ Department of Physics, University of Warwick, Coventry CV4 7AL, United Kingdom

${ }^{79}$ University of Wisconsin, Madison, Wisconsin 53706, USA

${ }^{80}$ Yale University, New Haven, Connecticut 06511, USA

(Received 18 July 2007; published 19 September 2007)

\footnotetext{
*Deceased

Now at Tel Aviv University, Tel Aviv, 69978, Israel

${ }^{\ddagger}$ Also with Università di Perugia, Dipartimento di Fisica, Perugia, Italy

${ }^{\S}$ Also with Università della Basilicata, Potenza, Italy

"Also with Universitat de Barcelona, Facultat de Fisica, Departament ECM, E-08028 Barcelona, Spain
} 
A measurement of the $\tau^{-} \rightarrow K^{-} \pi^{0} \nu_{\tau}$ branching fraction has been made using $230.2 \mathrm{fb}^{-1}$ of data recorded by the BABAR detector at the PEP-II $e^{+} e^{-}$collider, located at the Stanford Linear Accelerator Center (SLAC), at a center-of-mass energy $\sqrt{s}$ close to $10.58 \mathrm{GeV}$. We measure $\mathcal{B}\left(\tau^{-} \rightarrow K^{-} \pi^{0} \nu_{\tau}\right)=$ $(0.416 \pm 0.003$ (stat) $\pm 0.018($ syst $)) \%$.

DOI: 10.1103/PhysRevD.76.051104

PACS numbers: 13.35.Dx, 14.60.Fg, 11.30.Hv

The $\tau$ is the only lepton with a sufficiently large mass to decay to hadrons. Tau decays to hadronic final states proceed via $W$ exchange and thus the decay rates to the final states containing a strange quark are suppressed by the factor $\left(\left|V_{u s}\right| /\left|V_{u d}\right|\right)^{2}$ relative to the nonstrange final states, where $\left|V_{u d}\right|$ and $\left|V_{u s}\right|$ are the moduli of the Cabibbo-Kobayashi-Maskawa (CKM) matrix [1,2] elements. For a given value of $m_{s}[3],\left|V_{u s}\right|$ can be determined up to unprecedented precision [4,5] from the inclusive sum of the branching fractions of $\tau$ decays to hadronic final states with net strangeness equal to unity. This determination of $\left|V_{u s}\right|$ can be performed even without detailed knowledge of the hadronic mass spectrum arising due to incompleteness in our understanding of some of the intermediate resonance contributions.

In this paper we present a measurement of the $\tau^{-} \rightarrow$ $K^{-} \pi^{0} \nu_{\tau}$ branching fraction. ${ }^{1}$ In recent years, measurements of the branching fractions for $\tau$ decays to strange hadronic final states have been made using CLEO [6], ALEPH [7], and OPAL [8] data, but have often been limited by the size of the available data samples. The high luminosity provided by the PEP-II asymmetricenergy $e^{+} e^{-}$storage rings [9] at the Stanford Linear Accelerator Center (SLAC) coupled with the large cross section for $\tau^{+} \tau^{-}$-pair production has given us a very large sample for studying such decays in the $B A B A R$ detector.

The $B A B A R$ detector is described in detail elsewhere [10]. Charged particles are detected and their momenta measured with a five-layer double-sided silicon vertex tracker (SVT) and a 40-layer drift chamber (DCH) inside a $1.5 \mathrm{~T}$ superconducting solenoidal magnet. A ringimaging Cherenkov detector (DIRC) provides additional separation power for identification of charged particles for momenta greater than $1 \mathrm{GeV} / c$, and thus complements $\mathrm{d} E / \mathrm{d} x$ measurements in the $\mathrm{DCH}$, useful for the identification of charged particles below $1 \mathrm{GeV} / c$. Energies of photons and electrons are measured by a CsI(Tl) crystal electromagnetic calorimeter (EMC), and the instrumented magnetic flux return (IFR) is used to identify muons.

The analysis described in this paper is based on a data sample corresponding to an integrated luminosity of $208.7 \mathrm{fb}^{-1}$ collected at a center-of-mass energy $\sqrt{s}$ of $10.58 \mathrm{GeV}$ and $21.5 \mathrm{fb}^{-1}$ at $\sqrt{s}=10.54 \mathrm{GeV}$. With a cross section for $\tau^{+} \tau^{-}$pair production averaged over $\sqrt{s}$ of

\footnotetext{
${ }^{1}$ Throughout this paper, the charge conjugate decays are implied.
}

$\sigma_{\tau \tau}=(0.919 \pm 0.003) \mathrm{nb}$ [11], this total data sample of $230.2 \mathrm{fb}^{-1}$ contains $211.6 \times 10^{6} \tau^{+} \tau^{-}$pairs.

Studies of Monte Carlo (MC) simulated events are carried out for signal and various background samples; $\tau$ pairs are generated with $\operatorname{KK} 2 \mathrm{f}$ [12] and their decays simulated with TAUOLA [13]. Signal $\tau$ decays are modeled using form factors from the Breit-Wigner line shape of $K^{*}(892)$ decays [14], which nearly saturates the $\tau^{-} \rightarrow$ $K^{-} \pi^{0} \nu_{\tau}$ final state [6-8]. The other $\tau$ decays into any of the possible final states, according to the measured branching fractions [14]. To estimate non-tau backgrounds, samples of $Y(4 S) \rightarrow B \bar{B}, e^{+} e^{-} \rightarrow q \bar{q}(q=u, d, s, c)$, and $e^{+} e^{-} \rightarrow \mu^{+} \mu^{-}(\gamma)$ are generated with EvtGen [15], Jetset7.4 [16], and KK2f [12] MC programs, respectively. The available $\mathrm{MC}$ samples are weighted according to their respective size and cross sections in order to match the data integrated luminosity [14].

Each event is divided into hemispheres in the center-ofmass (CM) frame using the plane perpendicular to the thrust axis, which is the direction that maximizes the sum of the longitudinal components of the momenta of reconstructed particles, both neutral and charged. Only events with one charged track in each hemisphere, and with both tracks consistent with originating from the interaction point (1-1 topology) are selected. The net charge of the event is required to be zero.

To suppress light quark $(u, d, s)$ hadronic events, while retaining the relatively large fraction $(\approx 35 \%)$ of $\tau$ leptons that decay leptonically, we require that one hemisphere contains a track that is identified either as an electron $(e$-tag) or as a muon ( $\mu$-tag). The charged track in the opposite hemisphere is then required to be within the geometrical acceptance of the DIRC and to be identified as a kaon, and inconsistent with an electron. In order to reject events where the kaon has decayed or interacted before reaching the DIRC, a two-dimensional requirement on the Cherenkov angle $\left(\theta_{C}\right)$ versus the laboratory momentum of the candidate kaon $\left(p_{\text {lab }}\right)$ is used: $\theta_{C}(\mathrm{rad}) \leq$ $0.48+0.31 * p_{\text {lab }}(\mathrm{GeV} / c)$.

Event shape variables are used to discriminate against remaining $B \bar{B}$ and $q \bar{q}$ backgrounds. The thrust magnitude is required to be greater than 0.9 , and the ratio of the 2 nd to the 0th Fox-Wolfram moments [17] is required to be greater than 0.5. Also, to account for the substantial energy carried away by neutrinos in $\tau$ pair events, the total missing momentum in the laboratory frame is required to be greater than $0.5 \mathrm{GeV} / c$. This discriminates against Bhabha scattering and $\mu$-pair events, as well as $q \bar{q}$ production. 
Moreover, we remove events in which a $K_{S}^{0}$ decay to two charged pions is identified.

We further require that the event contains only one $\pi^{0}$. Only $\pi^{0}$ mesons that have been reconstructed from two separate EMC clusters with an energy above $50 \mathrm{MeV}$, not associated with charged tracks, are considered in the analysis. Candidate $\pi^{0}$ mesons are required to have an invariant mass in the range $100<m_{\gamma \gamma}<160 \mathrm{MeV} / c^{2}$ and an energy above $200 \mathrm{MeV}$ in the $\mathrm{CM}$ frame. The mass of the selected photon pair is constrained to the nominal $\pi^{0}$ mass [14] to improve the purity of the selected $\pi^{0} s$. The angle between the charged kaon and $\pi^{0}$ momenta in the CM frame is required to be less than $1.0 \mathrm{rad}$.

After all the above requirements, there remain 44348 $e$-tagged events, and $33764 \mu$-tagged events.

The MC simulated events are adjusted to improve their accuracy in modeling data events, according to several dedicated studies on specific control samples. Charged tracks are weighted to compensate for the different particle identification (PID) efficiencies between data and MC. On average, the MC efficiency is reduced by $15 \%$ and 3\% for muons and kaons, respectively. The electron identification is properly simulated and therefore no MC efficiency correction is applied. A correction to the $\pi^{0}$ MC efficiency has been obtained from detailed studies based on $\tau^{-} \rightarrow$ $\rho^{-} \nu_{\tau}$ and $\tau^{-} \rightarrow \pi^{-} \nu_{\tau}$ events. As a result of this study, the $\pi^{0}$ MC efficiency is reduced by $2 \%$.

We estimate the $\tau^{-} \rightarrow K^{-} \pi^{0} \nu_{\tau}$ selection efficiencies to be $1.31 \%, 0.96 \%$, and $2.27 \%$ for the $e$-tag, $\mu$-tag, and combined samples, respectively, using the signal MC sample with all requirements and corrections applied. The efficiency as a function of the $K^{-} \pi^{0}$ mass is consistent with being constant.

Figure 1 shows the invariant mass spectra of the selected $K^{-} \pi^{0}$ candidates and simulated backgrounds for the combined sample after all the analysis requirements. The contribution from light quarks, $c \bar{c}$ and $\mu^{+} \mu^{-}(\gamma)$ backgrounds is much smaller than the $\tau$-pair backgrounds. The contribution from $B \bar{B}$ backgrounds is negligible. The $K^{*}(892)^{-}$ resonance is seen prominently above the simulated background. Decays to higher $K^{*}$ resonances are expected near $1.4 \mathrm{GeV} / c^{2}$ [18-20], such as the $K^{*}(1410)^{-}$[21] and $K_{0}^{*}(1430)^{-}$[21], but their branching fractions are not well measured yet. These decays are not included in our simulation of $\tau$-pair events, but appear to be present in the data near $1.4 \mathrm{GeV} / c^{2}$. Below $0.7 \mathrm{GeV} / c^{2}$, the background is dominated by $K^{-} \pi^{0} \pi^{0}$ and $K^{-} K^{0} \pi^{0}$ events, for which the branching fractions are only known with relative uncertainties $\approx 40 \%$ and $\approx 15 \%$, respectively. These uncertainties are taken into account in the estimation of the systematic uncertainty due to modeling of the $\tau$ backgrounds.

The branching fraction $\mathcal{B}\left(\tau^{-} \rightarrow K^{-} \pi^{0} \nu_{\tau}\right)$ is calculated as $\mathcal{B}=\left[N_{\text {sel }}-N_{\text {bkg }}\right] / 2 \varepsilon_{\text {sig }} N_{\tau \tau}$, where $N_{\tau \tau}=\mathcal{L} \quad \sigma_{\tau \tau}=$ $2.116 \times 10^{8}$ is the number of produced $\tau^{+} \tau^{-}$pairs; $\varepsilon_{\text {sig }}$
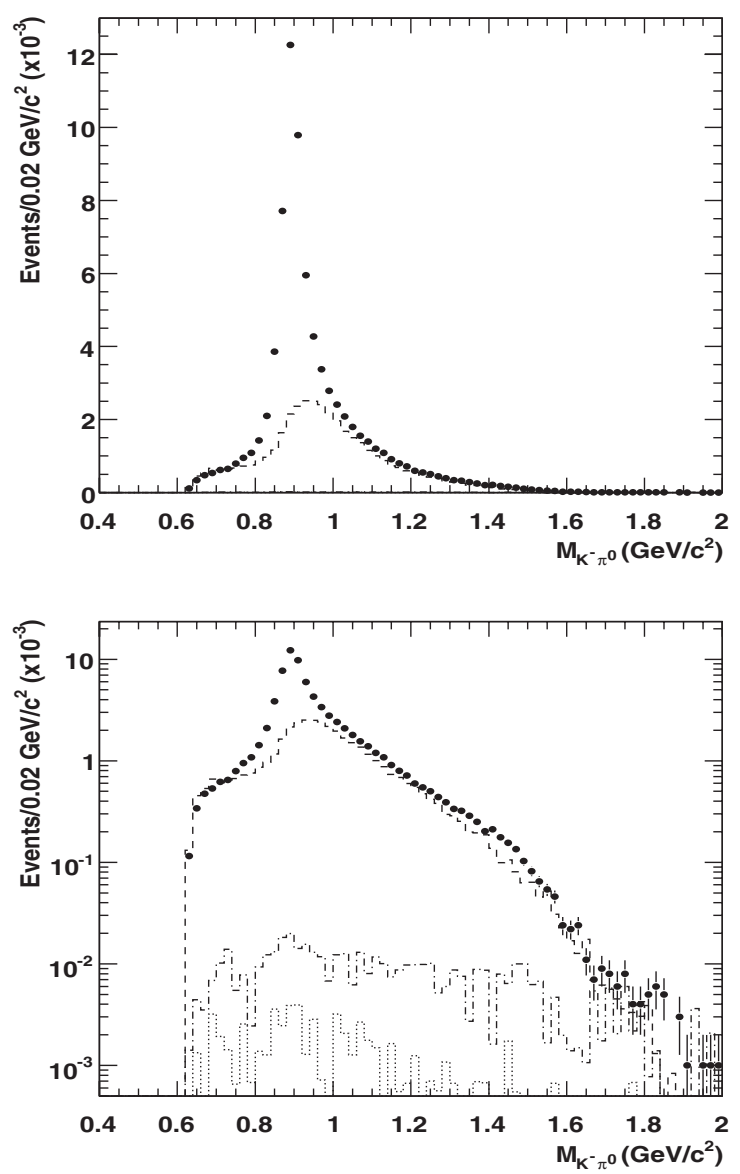

FIG. 1. Distribution of the reconstructed $K^{-} \pi^{0}$ mass for the combined sample in linear (top) and logarithmic (bottom) scale. The dots are the data, while the histograms are background MC events with selection and efficiency corrections: $\tau$ background (dashed line), $q \bar{q}$ (dash-dotted line), $\mu^{+} \mu^{-}$(dotted line).

is the estimated signal selection efficiency; $N_{\text {sel }}$ is the number of events selected in data; and $N_{\mathrm{bkg}}$ is the estimated number of background events obtained from MC simulations. For the combined $e$-tagged and $\mu$-tagged sample, $N_{\text {sel }}=78112 \pm 280$ events and the estimated background is $N_{\text {bkg }}=38247 \pm 159$ events. The branching fraction $\mathcal{B}\left(\tau^{-} \rightarrow K^{-} \pi^{0} \nu_{\tau}\right)$ is found to be $(0.416 \pm 0.003$ (stat) $) \%$, where the statistical uncertainty comes from the uncertainty in the number of signal events, $N_{\text {sel }}-N_{\text {bkg }}$. Several cross checks were performed by determining the branching fraction as a function of tag type, $\pi^{0}$ momentum, kaon charge, and run period; all were found to give consistent results.

A summary of the systematic uncertainties is given in Table I. The uncertainty in the charged track reconstruction efficiency is estimated to be $0.31 \%$ per track, based on studies of data control samples of $\tau$-pair events decaying to one charged particle on one side and three charged particles on the other side. The systematic uncertainty associated with the efficiency of detecting a $\pi^{0}$ is $3.26 \%$. The stated uncertainty in the charged particle identification 
B. AUBERT et al.

TABLE I. Relative systematic uncertainties in the $\tau^{-} \rightarrow$ $K^{-} \pi^{0} \nu_{\tau}$ analysis.

\begin{tabular}{lccc}
\hline \hline \multicolumn{1}{c}{ Systematic } & $e$-tag & $\mu$-tag & Combined \\
\hline & $(\%)$ & $(\%)$ & $(\%)$ \\
Tracking efficiency & 0.62 & 0.62 & 0.62 \\
$\pi^{0}$ efficiency & 3.26 & 3.26 & 3.26 \\
Particle identification & 2.09 & 2.34 & 2.17 \\
$\tau \tau$ cross section $\left(\sigma_{\tau \tau}\right)$ & 0.31 & 0.31 & 0.31 \\
Luminosity $(\mathcal{L})$ & 0.94 & 0.94 & 0.94 \\
Signal modeling and MC statistics & 0.38 & 0.52 & 0.35 \\
$\tau$ backgrounds & 1.35 & 1.35 & 1.35 \\
TOTAL & 4.28 & 4.42 & 4.32 \\
\hline \hline
\end{tabular}

efficiency represents the combined uncertainty for the two charged tracks, $(e, K)$ or $(\mu, K)$. This uncertainty includes a contribution due to the misidentification of charged pions as kaons. The uncertainty associated with the $\tau^{+} \tau^{-}$pair production cross section is $0.31 \%$ [11] and the luminosity determination uncertainty is $0.94 \%$. The effects of approximations in the $\mathrm{MC}$ signal modeling and of the finite $\mathrm{MC}$ statistics on the overall efficiency are negligible, but have been included in the estimation of the systematic uncertainty. The branching fractions for several $\tau$ decay modes that contribute to the background, particularly Cabibbosuppressed decays, are not well known. The resulting uncertainty due to the $\tau$-pair background estimate on the $\tau^{-} \rightarrow K^{-} \pi^{0} \nu_{\tau}$ branching fraction is $1.35 \%$. Backgrounds from other sources are very small and their impact of the signal extraction is negligible.

The total systematic uncertainty is the quadratic sum of the individual sources described above, and is $4.32 \%$ for the combined sample.
PHYSICAL REVIEW D 76, 051104(R) (2007)

In summary, using $211.6 \times 10^{6} \tau^{+} \tau^{-}$pairs recorded by the $B A B A R$ detector, we obtain the following result:

$\mathcal{B}\left(\tau^{-} \rightarrow K^{-} \pi^{0} \nu_{\tau}\right)=(0.416 \pm 0.003$ (stat) \pm 0.018 (syst $\left.)\right) \%$.

This measurement of the branching fraction is the most precise to date and is consistent with the existing world average, $\mathcal{B}\left(\tau^{-} \rightarrow K^{-} \pi^{0} \nu_{\tau}\right)=(0.454 \pm 0.030) \%$ [14].

We are grateful for the extraordinary contributions of our PEP-II colleagues in achieving the excellent luminosity and machine conditions that have made this work possible. The success of this project also relies critically on the expertise and dedication of the computing organizations that support $B A B A R$. The collaborating institutions wish to thank SLAC for its support and the kind hospitality extended to them. This work is supported by the U.S. Department of Energy and National Science Foundation, the Natural Sciences and Engineering Research Council (Canada), the Commissariat à l'Energie Atomique and Institut National de Physique Nucléaire et de Physique des Particules (France), the Bundesministerium für Bildung und Forschung and Deutsche Forschungsgemeinschaft (Germany), the Istituto Nazionale di Fisica Nucleare (Italy), the Foundation for Fundamental Research on Matter (The Netherlands), the Research Council of Norway, the Ministry of Science and Technology of the Russian Federation, Ministerio de Educación y Ciencia (Spain), and the Science and Technology Facilities Council (United Kingdom). Individuals have received support from the Marie-Curie IEF program (European Union) and the A.P. Sloan Foundation.
[1] N. Cabibbo, Phys. Rev. Lett. 10, 531 (1963).

[2] M. Kobayashi and T. Maskawa, Prog. Theor. Phys. 49, 652 (1973).

[3] M. Jamin, J. A. Oller, and A. Pich, Phys. Rev. D 74, 074009 (2006).

[4] E. Gamiz, M. Jamin, A. Pich, J. Prades, and F. Schwab, J. High Energy Phys. 01 (2003) 060.

[5] K. Maltman and C.E. Wolfe, arXiv:hep-ph/0703314.

[6] M. Battle et al. (CLEO Collaboration), Phys. Rev. Lett. 73, 1079 (1994).

[7] R. Barate et al. (ALEPH Collaboration), Eur. Phys. J. C 10, 1 (1999).

[8] G. Abbiendi et al. (OPAL Collaboration), Eur. Phys. J. C 35, 437 (2004).

[9] PEP-II collaboration, SLAC, Report No. SLAC-418 1993.

[10] B. Aubert et al. (BABAR Collaboration), Nucl. Instrum. Methods Phys. Res., Sect. A 479, 1 (2002).
[11] S. Banerjee, B. Pietrzyk, J.M. Roney, and Z. Was, arXiv:0706.3235.

[12] B. F. L. Ward, S. Jadach, and Z. Was, Nucl. Phys. B, Proc. Suppl. 116, 73 (2003).

[13] S. Jadach, Z. Was, R. Decker, and J.H. Kühn, Comput. Phys. Commun. 76, 361 (1993).

[14] W.-M. Yao et al. (Particle Data Group), J. Phys. G 33, 1 (2006).

[15] D. J. Lange, Nucl. Instrum. Methods Phys. Res., Sect. A 462, 152 (2001).

[16] T. Sjöstrand, Comput. Phys. Commun. 82, 74 (1994).

[17] G. C. Fox and S. Wolfram, Nucl. Phys. B149, 413 (1979).

[18] M. Finkemeier and E. Mirkes, Z. Phys. C 72, 619 (1996).

[19] K. Maltman, Nucl. Phys. B, Proc. Suppl. 123, 149 (2003).

[20] E. Gamiz, M. Jamin, A. Pich, J. Prades, and F. Schwab, Phys. Rev. Lett. 94, 011803 (2005).

[21] R. Barate et al. (ALEPH Collaboration), Eur. Phys. J. C 11, 599 (1999). 